\title{
Le massif forestier du dehors et du dedans : limites, marges et discontinuités de l'espace forestier
}

Forest clumps of the outside and the inside: limits, margins and discontinuities

of forest space

Das Forstmassiv des äußeren und des Inneren: Grenzen, Spielräume und

Unstetigkeiten des Forstraums

\section{Marc Galochet}

\section{OpenEdition}

\section{Journals}

Édition électronique

URL : http://journals.openedition.org/rge/1917

DOI : $10.4000 /$ rge. 1917

ISSN : 2108-6478

\section{Éditeur}

Association des géographes de l'Est

Édition imprimée

Date de publication : 1 janvier 2009

ISSN : 0035-3213

\section{Référence électronique}

Marc Galochet, « Le massif forestier du dehors et du dedans : limites, marges et discontinuités de l'espace forestier », Revue Géographique de l'Est [En ligne], vol. 49 / 2-3 | 2009, mis en ligne le 11 octobre 2010, consulté le 08 septembre 2020. URL : http://journals.openedition.org/rge/1917 ; DOI : https://doi.org/10.4000/rge.1917

Ce document a été généré automatiquement le 8 septembre 2020

Tous droits réservés 


\section{Le massif forestier du dehors et du dedans : limites, marges et discontinuités de l'espace forestier}

Forest clumps of the outside and the inside: limits, margins and discontinuities

of forest space

Das Forstmassiv des äußeren und des Inneren: Grenzen, Spielräume und

Unstetigkeiten des Forstraums

Marc Galochet

\section{Introduction : "Le dedans ou le dehors ? »}

1 Comment le géographe pourrait-il étudier les espaces forestiers en n'ayant qu'une seule vision de la forêt, celle de l'intérieur, celle du dedans? Cette vision resterait bien tronquée pour décrire et comprendre l'organisation des peuplements forestiers, dans leur diversité, leurs variations et leurs contrastes, à l'échelle d'un massif forestier ou à celle d'une parcelle. Il est nécessaire de sortir de la forêt pour mieux l'étudier et avoir aussi une vision de l'extérieur pour mieux l'appréhender.

2 C'est précisément cette démarche que certains géographes ont développé pour appréhender la forêt dans toutes ses dimensions à la fois « du dedans » et « du dehors » en confrontant les informations recueillies à chaque niveau, de la petite à la grande échelle (Rougerie, 1985 ; Hotyat, 1990 ; Galochet, 2002b). Pour Micheline Hotyat, il s'agit «d'acquérir une connaissance statistique de la forêt à l'échelle nationale, puis d'en déceler des structures et des distributions spatiales à l'échelle régionale, enfin d'en approcher les structures internes, le fonctionnement et les dynamiques à l'échelle locale »(Hotyat, 1990, p. 11).

3 Le dedans et le dehors sont alors deux approches nécessaires et complémentaires pour comprendre la diversité paysagère et végétale des espaces forestiers. Comme l'évoque Gabriel Rougerie, cette opposition entre l'extérieur et l'intérieur est un faux débat dans 
la mesure où «le paysage est si riche et si complexe que son étude exige une appréhension à la fois du dessus et du dedans » et par ailleurs, il estime que "la connaissance de l'intérieur complète l'approche scientifique traditionnelle abordée de l'extérieur » (Rougerie, 1985). Le passage de l'extérieur vers l'intérieur doit se faire par une approche méthodologique maîtrisant tous les niveaux scalaires de la démarche emboîtée pour l'analyse du paysage forestier, notamment au moyen de l'image satellitale ou du LiDAR ${ }^{1}$ pour appréhender l'extérieur, et par l'emploi de la photographie aérienne pour guider les relevés de terrain et pénétrer l'intérieur des espaces forestiers. A chaque changement d'échelle doit intervenir un concept géographique adapté comme ceux du sylvosystème et du sylvofaciès développés par Gérard Houzard (1980) permettant d'affiner l'étude des espaces forestiers (Galochet, 2006).

Cependant, avant d'aborder la question des concepts et des échelles géographiques d'analyse de l'espace forestier, encore faut-il lui trouver une définition claire pour identifier l'objet d'étude, ce qui constitue une première difficulté. Si la définition même $\mathrm{du}$ terme forêt est multiple, polysémique, complexe et sujette à controverses, que dire alors pour celle du massif forestier. L'un comme l'autre tiennent compte de la surface, de la densité, de la hauteur des arbres et du taux de recouvrement. A partir de ses éléments de définition les plus courants, on peut considérer qu'un massif forestier correspond à une vaste étendue boisée, relativement dense, constituée d'un ou de plusieurs peuplements d'arbres selon le découpage parcellaire de gestion et selon les types de traitements sylvicoles menés.

5 En France, l'Inventaire forestier national (IFN) est l'organisme de référence de recensement permanent des ressources forestières de la métropole qui définit la forêt comme « des formations boisées de production des formations végétales comprenant des arbustes appartenant à des essences forestières qui satisfont aux conditions suivantes :

- soit être constituées de tiges recensables (diamètre à 1,30 mètre du sol égal ou supérieur à $7,5 \mathrm{~cm}$ ) dont le couvert apparent (projection de leur couronne au sol) est d'au moins $10 \%$ de la surface du sol ;

- soit présenter une densité à l'hectare d'au moins 500 jeunes tiges non recensables (plansrejets-semis), vigoureuses, bien conformées, bien réparties;

- avoir un surface d'au moins 5 ares avec une largeur en cime d'au moins 15 mètres ;

- ne pas avoir une fonction de protection ou d'agrément.

6 A l'intérieur de ces formations boisées, on distingue les massifs boisés d'au moins 4 hectares avec une largeur moyenne en cime d'au moins 25 mètres, des boqueteaux dont la superficie est comprise entre 50 ares et 4 hectares et les bosquets dont la surface ne doit pas dépasser 50 ares. » (IFN, 1986 in Arnould, 1991).

7 Une définition plus récente de l'IFN complète les critères sémantiques en stipulant que «La forêt est un territoire occupant une superficie d'au moins 50 ares avec des arbres capables d'atteindre une hauteur supérieure à 5 mètres à maturité in situ, un couvert arboré de plus de $10 \%$ et une largeur d'au moins 20 mètres. $»^{2}$. Une forêt regroupe donc les bois et les boqueteaux mais pas les bosquets inférieurs à 50 ares et inclut en outre les peupleraies longtemps classées à part.

8 Les critères de surface, de densité, de couvert sont donc des éléments fondamentaux dans la définition de la forêt pour l'IFN comme l'évoque Paul Arnould (1991), mais ils sont aussi des éléments de distinction et d'appréhension déterminants pour en faire 
une analyse géographique fondée sur la notion de territoire forestier permettant d'en comprendre toute la complexité des problématiques (Galochet, Glon, 2010 ; Simon, 2006).

9 Face à la complexité de la définition, de l'organisation multiforme de l'espace forestier et de son évolution au cours du temps, diverses questions liées au caractère discontinu et non-linéaire de certains processus de spatialité et de temporalité, de certaines situations ou certaines évolutions peuvent se poser.

Comment le continu et le discontinu de l'espace forestier s'articulent-ils? Sur quels critères fonder les limites de l'espace forestier? Comment s'organise la marge du massif forestier par rapport à l'espace limitrophe et comment évolue-t-elle? Cette marge forestière peut-elle être caractérisée de front, d'interface ou de rupture?

Autrement dit, les notions de limites et de marges ne sont pas seulement considérées comme des lignes de séparation marquant une discontinuité dans l'espace forestier mais des concepts de catégorisation et de différenciation permettant de saisir toute la complexité de l'espace forestier en tant qu'objet géographique façonné, transformé, parcellisé, exploité, protégé, aménagé par les hommes. Pour tenter de répondre à ces questions, nous allons aborder le massif forestier dans ses diverses dimensions, d'abord du dehors, puis du dedans et enfin par la marge.

\section{I - Le dehors du massif forestier : fragmentation et discontinuité paysagères}

"L'espace de notre vie n'est ni continu, ni infini,
ni homogène, ni isotrope. Mais sait-on
précisément où il se brise, où il se courbe, où il se
déconnecte et où il se rassemble? »
Georges Pérec, Espèces d'espaces. Editions Galilée, 1974

\section{A - Fragmentation et discontinuité de l'espace forestier}

Une couverture forestière est rarement continue et homogène compte tenu des conditions du milieu très variables et de la mise en valeur des terres par les sociétés rurales conduisant à des concurrences foncières au cours de l'histoire. La couverture forestière européenne illustre particulièrement bien l'extrême diversité de situations et de fragmentations liée à l'histoire forestière ${ }^{3}$ et au mode de propriété. La diversité de la couverture forestière tient à la taille des différentes formations (arbres épars, bosquets, boqueteaux, petits bois, îlots boisés, forêts) mais aussi à la place occupée par l'arbre dans une formation végétale (forêt dense, forêt claire, forêt ouverte...). Ce contraste de la physionomie paysagère de la couverture forestière est lié non seulement aux conditions du milieu (climat, sol, relief, exposition...) mais également à l'action pluriséculaire de l'homme qui tantôt a exercé une pression sur le territoire forestier, tantôt a relâché sa pression en fonction du contexte géopolitique, économique, historique.

La couverture forestière européenne porte les marques de cette histoire naturelle et humaine dans son organisation comme dans sa fragmentation qui peuvent être appréhendées à la fois par les méthodes de l'écologie du paysage, développée en France 
notamment par Jacques Baudry et Françoise Burel (Burel, Baudry, 1999), mais aussi par l'approche des discontinuités spatiales plus familière aux géographes (Alexandre, Génin, 2008 ; Brunet, 1968 ; Carroué et al., 2002 ; Gay, 1995).

14 La notion de discontinuité peut être envisagée à la fois comme une propriété présentant un caractère discontinu d'un espace, comme une forme spatiale articulant ou séparant deux espaces voisins et différents, ou encore comme une dynamique spatio-temporelle intervenant comme une rupture dans un mouvement ou dans une évolution. Ainsi, l'approche par les discontinuités permet de repérer, d'identifier et d'expliquer des ruptures dans la structuration spatiale mais aussi des transitions dans l'espace faisant intervenir des logiques de différenciation, de seuil ou encore d'interface (Lecompte, Alexandre, 1996). Cependant «Mettre en évidence les différenciations spatiales au sein du couvert végétal ne relève pas de la simple description mais bien de la volonté de mettre en relation des modifications physionomiques et floristiques avec des facteurs explicatifs d'ordre essentiellement écologique et/ou historique. » (Simon, 2002, p. 143). Dès lors qu'on considère que tout espace est hétérogène, il devient alors intéressant de comprendre comment se forme, se structure, fonctionne et évolue la discontinuité spatiale. Elle devient aussi d'une certaine manière limite et marge d'un espace.

Il s'agit alors de considérer la discontinuité comme une ligne de séparation et de contact entre des espaces bien différenciés et observables dans l'espace forestier, mais aussi comme un moyen de distinguer différentes catégories de construction de sylvofaciès évoluant dans le temps et dans l'espace.

\section{B - Fragmentation, discontinuité et insularité du massif forestier}

La couverture forestière est souvent fragmentée en de multiples morceaux de taille et de forme différentes dans un espace plus vaste rappelant le caractère insulaire des îlots boisés isolés dans une mer de champs qui constitue les agrosystèmes. Le degré de fragmentation de la couverture forestière par la mise en valeur agricole des sols conduit à une discontinuité dans les habitats et dans les équilibres écologiques. La fragmentation peut donner lieu à des configurations morphologiques et paysagères très différentes soit totalement isolées soit groupées en archipels distants de grands massifs forestiers comme dans l'exemple ci-dessous du Gâtinais occidental situé entre les massifs forestiers de Fontainebleau et d'Orléans au sud de la région parisienne (Figure 1). 
Figure 1 : La fragmentation du couvert forestier du Gâtinais occidental.

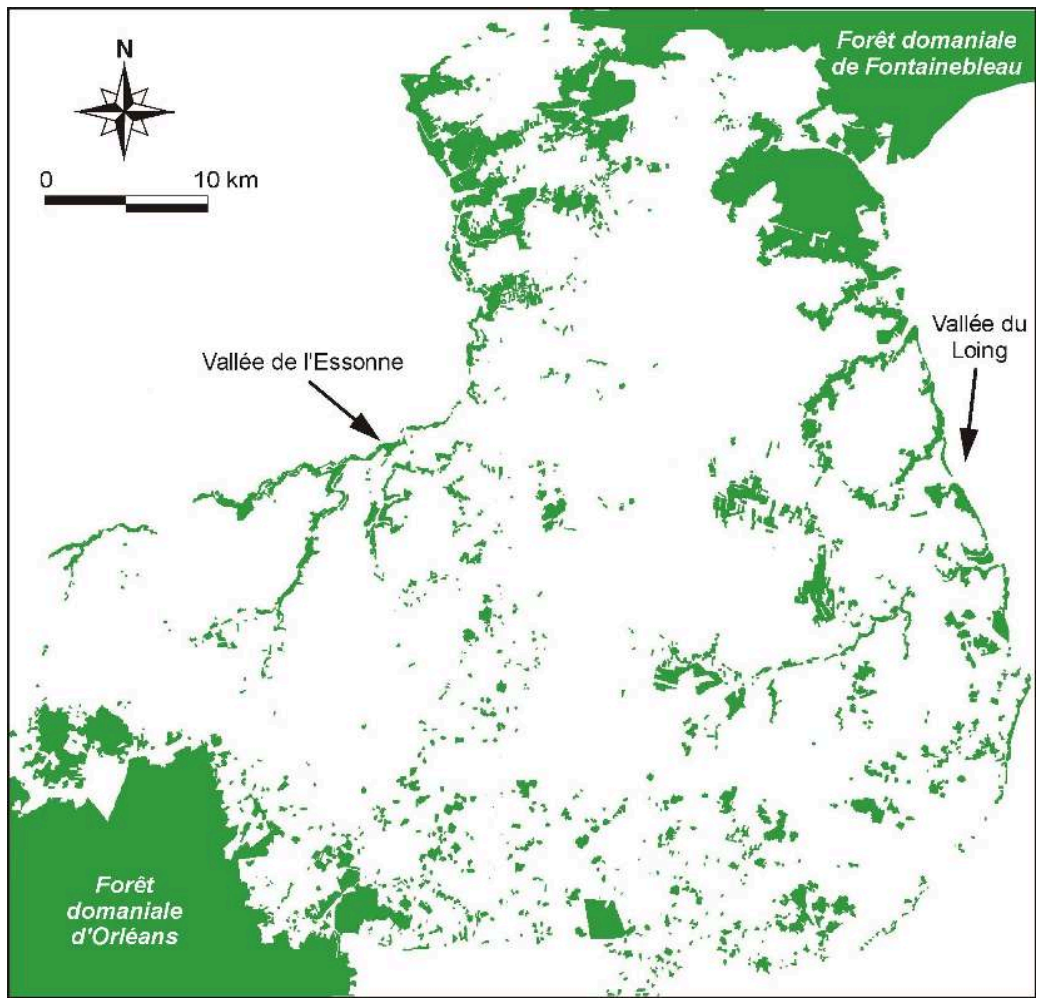

Document réalisé à partir des cartes topographiques IGN au 1/50 000 de Malesherbes, Fontainebleau, Pithiviers, Puiseaux, Château-Landon, Beaune-la-Rolande, Bellegarde, Lorris $(1986,1988)$.

Cette organisation spatiale et morphologique, où les espaces forestiers sont isolés et dispersés, renvoie à la théorie d'écologie des populations de biogéographie insulaire développée en 1963 par deux écologistes américains, Robert H. MacArthur et Edward O. Wilson, qui ont tenté de regrouper et d'ordonner toutes les informations accumulées sur les îles, dans une théorie quantitative relativement simple, permettant d'expliquer et de faire des prévisions sur la biogéographie insulaire: The theory of island biogeography publiée en 1967. A partir d'observations sur la distribution des espèces dans les îles (en termes d'abondance d'individus pour une espèce), MacArthur et Wilson montrent que le peuplement, en nombre d'espèces, de toute île est le résultat d'un équilibre dynamique établi entre deux processus continus : l'immigration (qui apporte sans arrêt de nouvelles espèces) et l'extinction (disparition des espèces déjà installées).

Selon cette théorie, les risques de disparition dans une île sont d'autant plus élevés pour une petite population qu'elle est éloignée d'un continent source. Les principaux facteurs discriminant retenus sont donc la distance de l'île par rapport à sa source de colonisation et la taille de l'île. C'est-à-dire que plus l'île est éloignée d'un continent source, plus la population insulaire est pauvre, compte tenu des exigences écologiques de chacune des espèces, des concurrences exercées et de la distance à parcourir.

En transposant cette théorie de biogéographie insulaire aux espaces forestiers, il convient d'ajouter les données du milieu et de prendre en considération l'influence des actions humaines sur le milieu pour mieux comprendre leur complexité et leur biodiversité.

La fragmentation de la couverture forestière et son organisation spatiale créent des conditions d'isolement qui agissent sur la diversité végétale des îlots boisés. Si la 
théorie de l'équilibre dynamique permet d'expliquer la constitution et l'évolution des biocénoses insulaires par la conjonction de deux phénomènes, l'immigration des espèces nouvelles et l'extinction d'espèces, alors est-il possible de considérer la proximité d'un massif forestier comme une source permettant l'enrichissement d'espèces migrantes dans les îlots boisés environnants ? Inversement, est-ce que le taux d'extinction est plus important si les îlots boisés sont très isolés et distants d'un grand massif forestier?

Ces quelques interrogations poussent à compléter la théorie de biogéographie insulaire par l'approche de l'écologie du paysage pour considérer les espaces forestiers comme un réseau créant un maillage territorial plus ou moins structuré dans lequel on peut observer des transferts de populations liés à la proximité mais aussi à la connectivité par effet de corridors biologiques entre massifs forestiers ou structures boisées offrant une diversité d'habitats et de peuplements dans un espace forestier discontinu et fragmenté.

Les îlots boisés, dispersés et isolés dans l'organisation du parcellaire agricole des vastes paysages d'openfield, peuvent être envisagés comme des fragments d'écosystèmes forestiers, mais aussi comme des noyaux de biodiversité tant floristique que faunistique plus ou moins riches, derniers refuges de la biodiversité (Galochet, 2003).

\section{II - Le dedans du massif forestier : structure et biodiversité forestières}

\section{A - Structures foncières et forestières}

L'approche interne du massif forestier par l'observation de terrain permet de décrire les structures forestières qui révèlent des décisions individuelles ou des gestions publiques retenues par les forestiers au cours du temps, comme un empilement de passés multiples correspondant à des héritages sylvicoles (Galochet, 2002a et 2006). Les espaces forestiers accumulent la mémoire des coupes et sont un véritable conservatoire des pratiques sylvicoles, décelables dans les structures forestières, qui s'organisent en fonction du parcellaire, offrant ainsi une couverture forestière fortement diversifiée. Les diverses interventions et pratiques des propriétaires forestiers, conjuguées au rôle du parcellaire multitaille au sein du massif forestier comme dans l'exemple présenté cidessous, favorisent l'hétérogénéité structurale de la végétation (Figure 2).

Figure 2 : Un parcellaire forestier multitaille dans un bois privé du Gâtinais occidental (bois de Bouchereau).

Source: M. Hotyat, M. Galochet, 2001. 


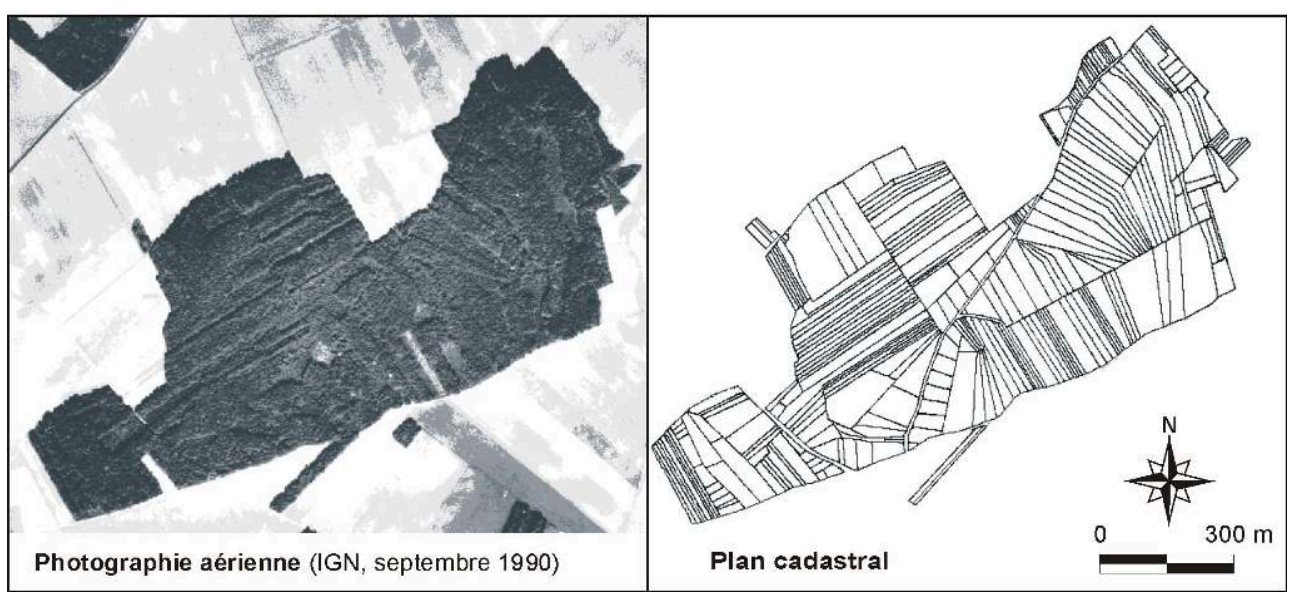

Cet exemple $\mathrm{du}$ bois de Bouchereau montre l'extrême diversité de la taille du parcellaire qui révèle le fort morcellement des massifs forestiers privés à la suite des nombreux partages successoraux au cours du temps. D'une superficie de 49 hectares, ce bois est morcelé en 289 parcelles, allant de $21 \mathrm{~m}^{2}$ à 1,23 hectare, réparties entre 166 propriétaires (Hotyat, Galochet, 2001).

Le découpage foncier des espaces forestiers, qui se matérialise par le parcellaire - c'està-dire l'ensemble des parcelles qui divise l'espace boisé -, engendre une mosaïque de peuplements issue des différentes activités sylvicoles pratiquées, et met en évidence des structures forestières diversifiées. Tantôt marqué, tantôt masqué, le parcellaire est parfois délimité par des bornes ou par des fossés. Par ailleurs, le parcellaire de gestion et le parcellaire foncier ne se superposent pas systématiquement; une parcelle de propriété peut être divisée en plusieurs parcelles d'exploitation, tandis qu'une parcelle d'exploitation peut recouvrir plusieurs parcelles cadastrales. La prise en compte des structures foncières et forestières permet de croiser des critères juridiques et écologiques pour former le maillage parcellaire de l'espace forestier afin de mieux penser son unité de gestion.

Les espaces forestiers sont transformés en une véritable mosaïque de parcelles nées de la mise en œuvre, dans le temps et dans l'espace, des pratiques des différents propriétaires, comme de l'absence totale d'intervention d'un certain nombre d'entre eux. Le contraste des modes de gestion et de taille des parcelles forestières concourent à la multiplicité des paysages intra-forestiers accrue par le dessin, la taille et l'agencement des parcelles. La parcellisation, poussée à l'extrême dans le cas des espaces forestiers privés, explique toute la complexité et l'hétérogénéité de la structure forestière et paysagère du massif forestier renforcées par le mode de gestion et d'exploitation. Ainsi, dans ce contexte fortement anthropisé, l'homme doit être considéré comme un véritable facteur de diversité en milieu forestier, dont les choix de gestion sylvicole sont encore visibles aujourd'hui dans la structuration et la stratification du massif forestier.

\section{B - Exploitation forestière et conséquences sur la biodiversité}

L'intervention du forestier et les travaux consécutifs se concluent le plus souvent par une ouverture plus ou moins importante, plus ou moins brusque du couvert. Ces opérationstransforment les conditions du milieu notamment par l'apport plus important de lumière jusqu'au sol, permettant l'arrivée d'héliophytes (essences de 
lumière), et conditionnent ainsi la dynamique de reconquête végétale à venir. En créant des percées dans la couverture végétale, l'homme diversifie les biotopes et permet à une flore héliophile de se développer dans une parcelle coupée, associée aux espèces de sous-bois, elles-mêmes déterminées par la plus ou moins grande densité du couvert forestier.

Facteur d'enrichissement de la diversité végétale des espaces forestiers, les coupes de bois intra-forestières fractionnent la couverture végétale en de nombreuses unités de taille variable, dont l'étendue et la forme des coupes sont le plus souvent liées au parcellaire foncier, entrainant une multitude de stades de développement décalés dans le temps de parcelle en parcelle, depuis les dernières coupes de bois jusqu'aux futaies mûres. Ces conditions créent une couverture forestière fortement diversifiée. La recolonisation végétale d'une parcelle boisée abattue est conditionnée par son environnement. Par exemple, une petite parcelle entourée par des peuplements denses et hauts, coupée récemment, bénéficie de peu de lumière. A l'inverse, un peuplement moins dense dont le sous-bois est dégagé, développe ses strates basses et sa flore herbacée s'il se trouve encadré de plusieurs coupes récentes. Des dynamiques forestières variées s'établissent alors. C'est dire que les travaux sylvicoles exercés sur des peuplements favorisent le maintien d'une certaine diversité végétale et structurale tant au cœur du massif forestier que sur sa marge.

\section{III - La marge du massif forestier : interface et rupture entre sylvosystème et agrosystème}

\section{A - Flux et reflux de la lisière forestière : espace d'interface fluctuant et fragile}

Espaces agricoles et espaces boisés sont deux systèmes écologiques aux conditions environnementales très différentes, qui réagissent entre eux et qui entretiennent des relations conflictuelles. L'agrosystème s'exprime par une exploitation intensive dans les champs, une rotation des cultures, un rythme de production selon le calendrier cultural, un cycle court, l'usage d'engrais, une sélection des espèces... alors que dans le sylvosystème, les cycles sont beaucoup plus longs pour la croissance des arbres. Ce sont donc deux territoires contrastés constitués d'un côté de cycles culturaux rapides et de l'autre des cycles sylvoculturaux lents, dont les pas de temps entrent en résonance.

L'association entre les espaces boisés et les espaces agricoles, leur équilibre, leur superficie, leur mode de gestion contribuent à jouer un rôle déterminant dans la préservation de la diversité biologique. Ainsi, les haies, les lisières forestières, les bois offrent des conditions favorables à de nombreuses espèces pouvant nicher, s'alimenter, se cacher, se réfugier... Ce sont de véritables écotones ou zones d'interface qui jouent un rôle important dans l'interconnexion des sylvosystèmes par la continuité entre les réservoirs de biodiversité en limitant le fractionnement des populations d'espèces animales et végétales (cf. théorie de l'écologie du paysage).

L'agrosystème exerce une concurrence, locale mais bien réelle, sur le sylvosystème et ses marges. En effet, l'intensification agricole par l'agrandissement des structures foncières agraires, le remembrement du parcellaire, les défrichements pour la mise en culture sont des phénomènes manifestes sur l'emprise du sylvosystème composé de 
petits bois et de haies (lorsqu'elles ont résisté). Selon les périodes de forte productivité agricole, l'intensification agricole a, dans bien des cas, abouti à l'arasement des haies et des bois (Dubois, 1983). Les espaces boisés dans l'agrosystème sont le résultat de la combinaison de phénomènes naturels et d'actions anthropiques au cours de la longue mise en place du paysage et des relations complexes engendrant des concurrences qui s'exercent notamment sur l'interface des deux systèmes, représentée par la lisière externe des bois (Galochet, 2004).

Si la lisière forestière peut être considérée comme une interface entre bois et champ, elle devient aussi une zone de transition entre sylvosystème et agrosystème évoluant dans le temps et dans l'espace en fonction des concurrences foncières exercées de part et d'autre. Elle peut être aussi considérée à la fois comme un " espace flou » et comme une "zone de mouvance aux conditions biogéographiques spécifiques " (Husson, 1984, p. 415), dans la mesure où la lisière forestière est un espace fluctuant et intensément exploité depuis fort longtemps puisqu'elle correspondait sous l'Ancien Régime à une partie du finage fréquemment visitée, ayant pour fonction première de fournir du bois de chauffe. A partir de l'exemple lorrain au XVIII e siècle, Jean-Pierre Husson (1984) faisant écho à l'article de Jean-Claude Bonnefont (1974), explique les fondements historiques des conflits d'usages de la lisière externe exposée aux pressions de l'agrosystème voisin qui ne sait pas encore intensifier la production sur les terres déjà défrichées :

« En position de contact, elle [lisière externe] était zone d'enjeux et de conflits nés des intérêts contraires opposant les possédants soucieux d'affirmer leur pleine propriété forestière, et les communautés attachées aux traditionnelles valeurs usagères. [...] Les délits sont multiples et revêtent une grande diversité tant dans les formes que dans les méthodes utilisées. [...] L'ensemble de ces délits a pour but d'accroître l'espace agricole et de créer de nouveaux champs et prés, aux dimensions souvent modestes ».

J.-P. Husson, 1984. « Les lisières forestières au XVIII ${ }^{e}$ siècle ». Revue Forestière Française, p. 415, 422, 423.

Les travaux de Jean-Pierre Husson montrent combien la lisière forestière lorraine est fragile, menacée et fluctuante, car elle est avant tout le prolongement de l'espace agricole. Elle correspond aussi aux espaces utilisés de façon momentanée et ponctuelle pour délimiter, de manière imparfaite parfois, l'agrosystème du sylvosystème. D'un autre point de vue, la lisière est une frontière mouvante pour la forêt, issue d'une lutte séculaire entre le défrichement par l'homme et le reboisement spontané. L'étude des lisières nécessite donc de mettre l'accent non sur les aspects statiques, mais sur le caractère dynamique des formations végétales.

La lisière forestière devient une limite foncière du champ ou de la forêt, véritable contact entre deux mondes. Entre deux coupes d'entretien, une reconquête végétale spontanée affecte les positions les plus avancées de la lisière sur le champ (l'ourlet). Ainsi, les lisières sont stabilisées lorsque l'agrosystème reste bien cultivé, et elles sont qualifiées de lisières de reboisement lorsque les friches dominent. De ce milieu biogéographique original, façonné par l'homme comme une limite entre deux systèmes, on voit l'épaisseur d'un tissu foisonnant où la lisière s'étoffe en manteau et en ourlet (Figure 3). Par la variabilité du pas de temps des interventions de l'homme sur les lisières forestières, un rajeunissement permanent ou au contraire une reconquête progressive avec des allées et venues, entretiennent une diversité de situations qui 
engendre une augmentation de la diversité floristique et faunistique, constituant ainsi un espace privilégié en matière de biotopes.

Figure 3 : Typologie des lisières forestières.

Source : M. Galochet, thèse de doctorat, 2001 in M. Hotyat, M. Galochet, 2001.

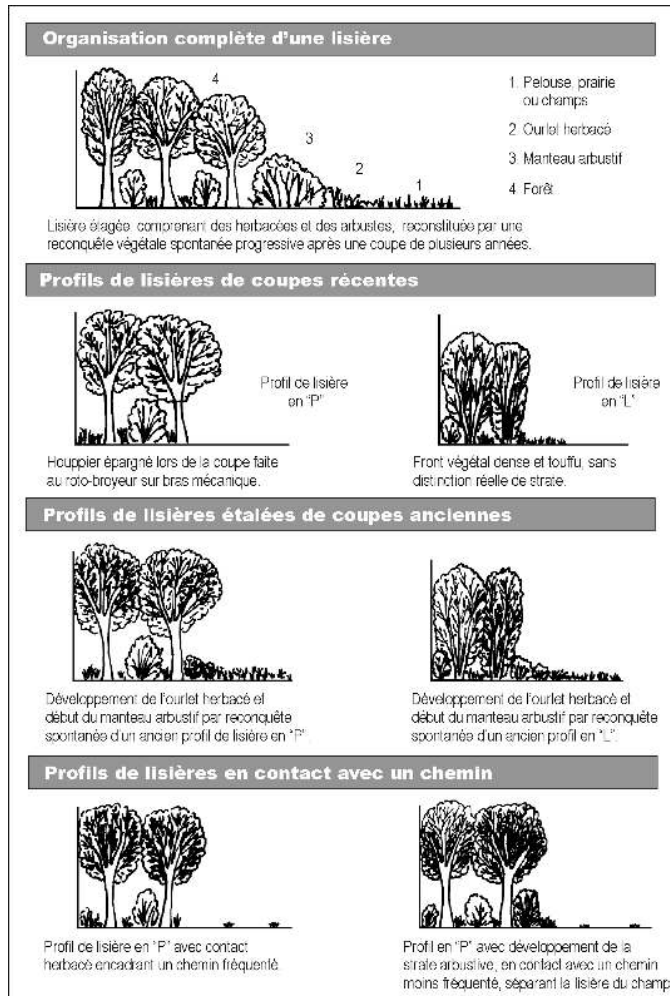

\section{$B$ - En marge du massif forestier : reconquête des terres agricoles abandonnées}

Pendant de nombreuses années, les agriculteurs français ont manqué de terres pour satisfaire les besoins d'agrandissement destinés à rentabiliser davantage leurs exploitations et pour répondre à la forte demande. La situation s'est aujourd'hui modifiée et varie considérablement en fonction des conditions locales.

Dans ce contexte agricole, les friches ont pu réapparaître, donnant naissance à une récente dynamique forestière accompagnée par des phénomènes de reboisement, spontané ou non, sur des parcelles agricoles abandonnées. Le temps de la friche constitue une période relativement fugace par rapport à la lente construction des sylvosystèmes et à l'élaboration de leurs aménagements. Jean-Pierre Husson associe Espaces de déprise et paysages flous (1999) à propos des deshérences du XVIIe siècle, et considère que « La friche, sortie du système de culture pour une période courte, longue, voire très longue [pouvant aboutir à la fruticée] est hirsute tout en laissant visible et reconnaissable l'ancienne utilisation: billons fossilisés, murettes et pierriers conservés,... La friche est un géosystème périforestier ou transitoire, une combinaison d'espaces confrontés à plusieurs échelles de temporalité qui peuvent se concrétiser par des successions lentes ou des évolutions brutales » (Husson, 1999, p. 76). 
37 La déprise agricole peut provoquer l'enfrichement des parcelles abandonnées, colonisées progressivement par des plantes vivaces (chardons, graminées, genêts...), puis les ronces, les ligneux de la fruticée (églantiers, prunelliers...), les arbres (bouleaux, saules, chênes, pins... selon les terrains), avant d'évoluer vers la lande boisée ou la forêt. A terme, la déprise agricole et le gel des terres, se traduisant soit par un abandon total de la parcelle, soit par une sous-utilisation, favorisent l'apparition de la friche qui facilite une reconquête forestière permettant la progression des espaces forestiers par leur périphérie et d'en augmenter leur superficie (Figure 4).

Figure 4 : Dynamiques forestières spontanées internes et externes.

Source: M. Galochet, thèse de doctorat, 2001.

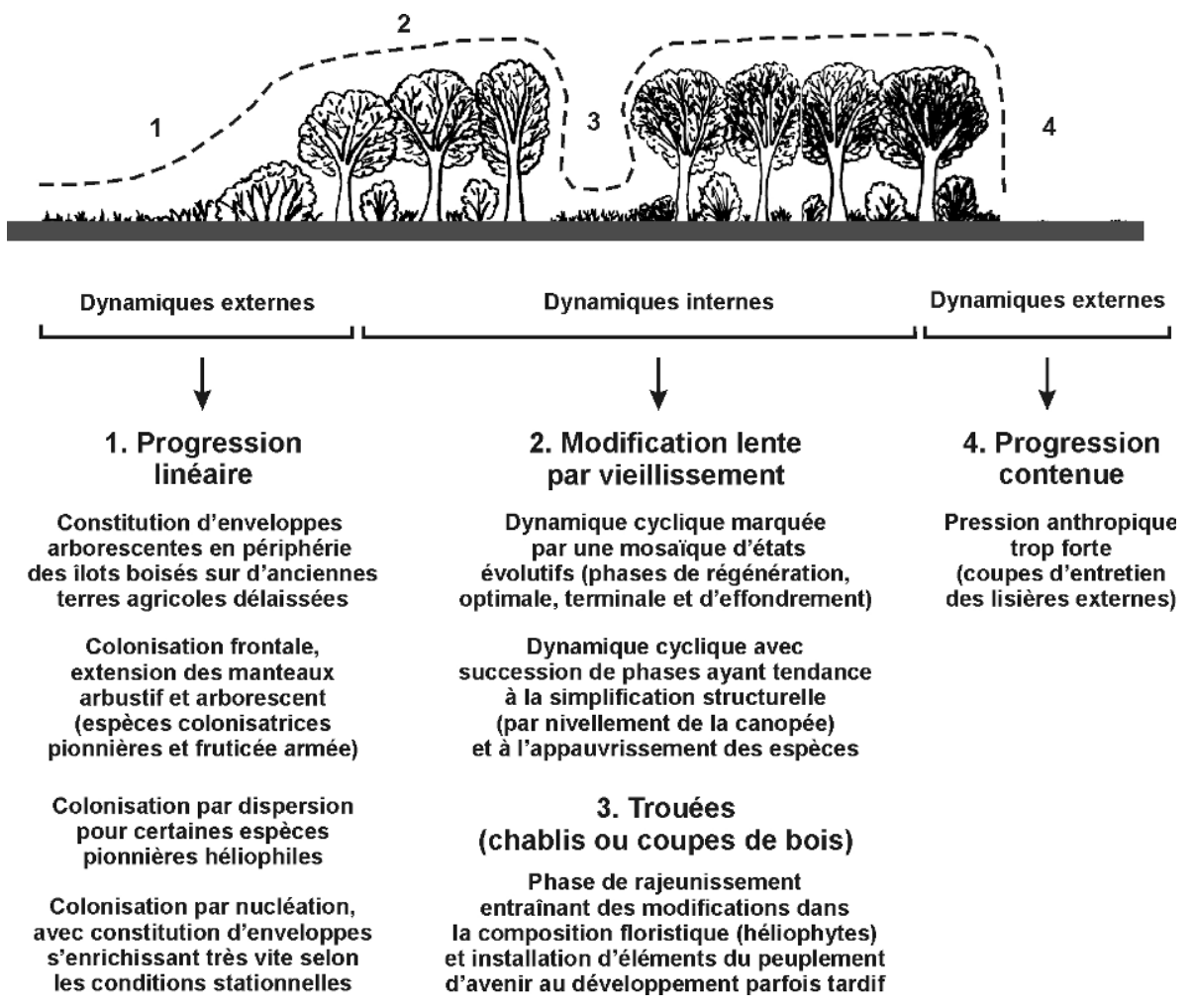

La progression forestière s'exerçant en périphérie des espaces boisés sur les terres agricoles attenantes abandonnéesse produit par dynamique spontanée dite linéaire, marquée par la présence d'espèces colonisatrices. Progressivement la constitution d'enveloppes arborescentes s'établit et aboutit à des successions forestières avec des stades pionniers, transitoires, débouchant sur un état optimal.

Les modes de reconquête du territoire par la végétation des espaces boisés (représentant la banque de semences), diffèrent selon le comportement des espèces et les activités anthropiques mises en oeuvre. On peut observer trois types de colonisation conduisant tous à l'extension péri-forestière :

- d'abord une colonisation frontale, liée à la simple extension, limitée, des manteaux arbustif et arborescent,

- ensuite une colonisation par dispersion pour certaines espèces pionnières héliophiles,

- enfin une colonisation par nucléation, avec constitution d'enveloppes s'enrichissant très vite, si les conditions stationnelles sont favorables. 


\section{des lisières forestières), alors la dynamique externe est contenue dans les limites} comprises entre l'espace boisé et l'espace agricole.

41 Si le gel des terres continue, il est probable que la superficie forestière de certaines régions augmentera aux dépens des terres agricoles délaissées, permettant par la même occasion aux îlots boisés et autres boisements sporadiques de se reconstituer et de reconquérir des positions perdues. Mais ces reboisements, souvent péri-forestiers, ne constitueront pas pour autant de grandes et belles forêts : c'est un paysage nouveau qui se crée, mi-agricole, mi-forestier, sur le pourtour des îlots boisés. Ces paysages nouveaux, issus de la réforme de la Politique Agricole Commune, permettent d'agrandir la périphérie des îlots boisés et deviennent de véritables zones de refuge pour la faune et la flore, participant ainsi à la qualité biologique et paysagère du territoire rural.

\section{Conclusion : Espaces forestiers et agricoles, des territoires imbriqués concurrents et complémentaires} espèces animales et végétales jouant un rôle non négligeable dans la préservation de la biodiversité souvent relativement importante à la fois dans l'espace forestier et à sa marge. A l'échelle du territoire, la discontinuité et la fragmentation de la couverture forestière permettent de comprendre le rôle fondamental dans la connexion des écosystèmes entre les différents réservoirs de biodiversité. La complémentarité des espaces jouent par conséquent en faveur de la diversité biologique avec la richesse des milieux mais participe aussi à la diversité paysagère qui s'inscrit dans un territoire comme le rappelle si justement Laurent Simon « La notion de territoire permet en effet d'entrer dans la complexité des problématiques liées à la biodiversité » (Simon, 2006, p. 452).

La limite entre l'espace forestier et l'espace agricole est loin d'être figée dans la mesure où les changements sont permanents; les deux espaces, considérés à la fois comme 
construit social, produit économique et ressource territoriale, connaissent des dynamiques entre permanences et mutations.

\section{BIBLIOGRAPHIE}

Alexandre F., Génin A. (dir.), 2008, Continu et discontinu dans l'espace géographique. Tours, Presses universitaires François Rabelais, Maison des Sciences de l'Homme «Villes et Territoires », 442 p. Arnould P. (1991) - « Forêts, nouvelles forêts et vieilles forêts ». A. Corvol (dir.), La Forêt, actes du $113^{\mathrm{e}}$ Congrès des Sociétés savantes, Strasbourg (5-9 avril 1988), Paris, CTHS, p. 13-30.

Bonnefont J.-C. (1974) - « Les lisières forestières en Lorraine : un milieu biogéographique original ». Revue Géographique de l'Est, 14, n¹-2, p. 3-29.

Brunet R. (1968) - Les phénomènes de discontinuité en géographie. Paris, CNRS éditions, coll. Mémoires et documents, vol. 7, $117 \mathrm{p}$.

Burel F., Baudry J. (1999) - Ecologie du paysage. Concepts, méthodes et applications. Paris, éditions Tec \& Doc, 359 p.

Carroué L. et al. (2002) - Limites et discontinuités en géographie. Paris, SEDES, coll. DIEM, 159 p.

Dubois J.-J. (1983) - « Les lisières forestières d'après les anciens plans des forêts de la région Nord au XVII et XVIII siècles ». Géhu J.-M. (éd.), Colloques Phytosociologiques VIII, « Les lisières forestières ", Vaduz, éditions J. Cramer, p. 273-285.

Galochet M. (2002a) - « Héritages sylvicoles dans les forêts et les îlots boisés de Grande Pologne ». Annales de Géographie, n623, p. 60-72.

Galochet M. (2002b) - « Du dessus au dedans : une approche biogéographique emboîtée des îlots boisés ». L'Information géographique, vol. 66, n4 4 p. 341-350.

Galochet M. (2003) - « Des bois dans les campagnes européennes, derniers refuges de la biodiversité ». La campagne colonisée. Cahiers Kubaba, nVI,L'Harmattan, p. 37-58.

Galochet M. (2004) - «Les lisières forestières ». Mercier D. et al., Le commentaire de paysages en géographie physique. Paris, Armand Colin, p. 164-167.

Galochet M. (dir.) (2006) - La forêt, ressource et patrimoine. Paris, Ellipses, coll. Carrefours, 272 p.

Galochet M., Glon E. (dir.) (2010) - Des milieux aux territoires forestiers. Arras, éditions Artois Presses Université, coll. Géographie, (à paraître).

Gay J.-C. (1995) - Les discontinuités spatiales. Paris, Economica, coll. Géo-poche, 112 p.

Hotyat M. (1990) - De l'espace territorial à l'analyse stationnelle : recherche méthodologique pour une approche biogéographique de la forêt française. Habilitation à diriger des recherches, Université Paris-VII, 298 p.

Hotyat M., Galochet M., Liège F. (1997) - « Petits bois et leurs lisières dans les plaines de grande culture : "entre nature et culture". Exemple pris dans le Gâtinais occidental ». Burnouf J., Bravard J.-P., Chouquer G. (éd.), La dynamique des paysages protohistoriques, antiques, médiévaux et modernes, Sophia Antipolis, éditions APDCA, p. 493-504. 
Hotyat M., Galochet M. (2001) - « L'homme, facteur de diversité en milieu forestier ». Bulletin de l'Association de Géographes Français (BAGF), n²001-2, p. 151-163.

Husson J.-P. (1984) - « Les lisières forestières au XVIII ${ }^{\mathrm{e}}$ siècle : l'exemple lorrain ». Revue Forestière Française, XXXVI-5, p. 415-424.

Husson J.-P. (1999) - « Espaces de déprise et paysages flous dans la Lorraine rurale à l'aube du XVIII ${ }^{\mathrm{e}}$ siècle ». Lotharingia, IX, p. 73-81.

Gilg O. (2003) - « La fragmentation des paysages forestiers : un problème pour la conservation de la biodiversité ». Daniel Vallauri (coord.), Livre Blanc sur la protection des forêts naturelles en France. Forêts métropolitaines, Paris, Editions Tec \& Doc, p. 87-94.

Lecompte M., Alexandre F. (1996) - « Discontinu et continu de la végétation et du milieu ». L'Espace géographique, vol. 25 (3), p. 261-272.

Rougerie G. (1985) - « Le dessus ou le dedans ? (approches paysagères) ». Revue Géographique de l'Est, n4, p. 355-363.

Simon L. (2002) - « De la plante à la formation végétale : les limites des découpages ». Carroué L. et al., Limites et discontinuités en géographie. Paris, SEDES, coll. DIEM, p. 141-150.

Simon L. (2006) - « De la biodiversité à la diversité : les biodiversités au regard des territoires ». Annales de Géographie, vol. 115, n651, p.451-467.

\section{NOTES}

1. Voir dans le même numéro l'article de Murielle Georges-Leroy, Jérôme Bock, Etienne Dambrine et Jean-Luc Dupouey.

2. Voir le site Internet de l'Inventaire Forestier National, http://www.ifn.fr

3. Voir les travaux du Groupe d'Histoire des Forêts Françaises, http://www.ghff.ens.fr

4. Voir dans le même numéro l'article de Claire Labrue.

\section{RÉSUMÉS}

La couverture forestière européenne est contrastée tant dans sa physionomie que dans sa gestion et ses usages. Ce contraste, lié à la fois à la fragmentation et à la discontinuité de l'espace forestier, empêche d'enfermer cet objet d'étude dans une approche scalaire univoque, et sa complexité réclame une vision à la fois du dehors et du dedans, à l'échelle du paysage et à celle du boisement, pour saisir toutes ses facettes biogéographiques et les facteurs explicatifs de sa biodiversité. Pour comprendre comment s'articule le continu et le discontinu de l'espace forestier et comment s'organise la marge du massif forestier par rapport à l'espace limitrophe, les notions de limites et de marges permettent une approche conceptuelle de catégorisation et de différenciation de l'espace forestier et d'en saisir toute sa complexité en tant qu'objet géographique territorialisé, exploité, protégé et aménagé par les hommes.

The European forest cover is contrasted as well in its aspect as in its management and its uses. This contrast, related at the same time on the fragmentation and the discontinuity of forest 
space, prevents from locking up this object of study in a univocal scalar approach, and its complexity claims a vision at the same time outside and inside, on a landscape scale and that of timbering, to seize all its biogeographic facets and the explanatory factors of its biodiversity. To understand how is articulated the continuous one and discontinuous forest space and how the margin of the main forest compared to space bordering is organized, the concepts of limits and margins allow a conceptual approach of categorization and differentiation of forest space and to seize of it all its complexity as a territorialized geographical object, exploited, protected and managed by the men.

Decken forstwirtschaftlich europäisch ist kontrastiert sowohl in sein Aussehen als auch in sein Verwaltungen und sein Gebräuche. Dieser an die Zerstückelung als auch an die Unstetigkeit des Forstraums sowohl gebundene Kontrast, verhindert, diesen Studiengegenstand in einem eindeutigen skalaren Konzept einzuschließen, und seine Komplexität fordert eine Vorstellung sowohl des äußeren als auch des Inneren im Maßstab der Landschaft und an jener der Aufforstung, um all seine biogeographischen Facetten und die erklärenden Faktoren seiner Artenvielfalt zu erfassen. Um zu begreifen, wie das kontinuierliche sich artikuliert, und das unterbrochene vom Forstraum und wie der Spielraum des Forstmassivs hinsichtlich des angrenzenden Raums sich organisiert, erlauben die Konzepte von Grenzen und von Spielräumen ein begriffliches Konzept der Kategorisierung und der Unterscheidung des Forstraums, und damit all seine Komplexität als geographischer Gegenstand, genutzter, geschützter und der von den Männern zu erfassen, eingerichtet wurde.

\section{INDEX}

Mots-clés : discontinuités, limites, marges, massif forestier

Schlüsselwörter : Forstmassiv, Grenzen, Spielräume, Unstetigkeiten

Keywords : discontinuities, forest clumps, limits, margins

\section{AUTEUR}

\section{MARC GALOCHET}

Maître de conférences en géographieUniversité Lille Nord de France (Artois)EA 2468 DYRT, F-62000 ArrasUMR 8185-CNRS ENEC Université Paris-Sorbonne 